\title{
Convexity Properties for a Function of Two Integer Variables
}

\author{
Sara Nourazari', Hillel Kumin² \\ ${ }^{1}$ Department of Health Care Administration, California State University, Long Beach, CA, USA \\ ${ }^{2}$ School of Industrial and Systems Engineering, University of Oklahoma, Norman, OK, USA \\ Email:sara.nourazari@csulb.edu,hkumin@ou.edu
}

How to cite this paper: Nourazari, S. and Kumin, H. (2021) Convexity Properties for a Function of Two Integer Variables. American Journal of Operations Research, 11, 253-256.

https://doi.org/10.4236/ajor.2021.116016

Received: September 9, 2021

Accepted: October 10, 2021

Published: October 13, 2021

Copyright ( 2021 by author(s) and Scientific Research Publishing Inc.

This work is licensed under the Creative Commons Attribution International License (CC BY 4.0).

http://creativecommons.org/licenses/by/4.0/ (c) (i) Open Access

\begin{abstract}
Sufficient conditions are given for any local minimum of a function of two integer variables to be a global minimum. An example is given to show that a function of two integer variables need not be discrete convex for this condition to hold.
\end{abstract}

\section{Keywords}

Linear Integer Programming, Convexity

\section{Introduction}

Integer programming problems occur in many real-life situations: production planning, scheduling, telecommunication networks, cargo loading, vehicle routing, and inventory maintenance, for example. There are numerous commercial software packages available for solving integer programming problems, such as CPLEX, Gurobi, KNITRO, and LINDO. There are also many open-source programs available. However, since the integer programming problem, in general, is NP-complete [1], it is very difficult to obtain a global optimum, and so, at best, a local optimum is generally found. For the case in which all of the decision variables are continuous, a sufficient condition for any local minimum to be a global minimum is that the Hessian matrix be positive definite [2]. If the objective function has two variables, one of which is integer and the other continuous, sufficient conditions for any local minimum to be a global minimum can be found in [3]. If the objective function has two integer variables, we give below sufficient conditions for any local minimum to be a global minimum. The function need not be integer convex for this condition to hold. 


\section{Two Integer Variables}

Definition 1: Let $g(z)$ be a real mapping from the integers into the real line. The first difference of $g(z)$ is denoted by $\Delta g(z)$ and is defined as follows:

$$
\Delta g(z)=g(z+1)-g(z)
$$

Second and subsequent difference of $g(z)$ are defined by

$$
\Delta^{r+1} g(z)=\Delta\left\{\Delta^{r} g(z)\right\}
$$

Definition 2: A function $g(z), z \in \mathbb{Z}$ is integer strictly convex if $\Delta g(z)$ is a strictly increasing function of $z$, that is, if $\Delta^{2} g(z)>0$.

Definition 3: The function $g(z), z \in \mathbb{Z}$ has a local minimum at $\bar{z}$ if $g(\bar{Z}+1) \geq g(\bar{z})$ and $g(\bar{z}-1) \geq g(\bar{z})$.

Definition 4: The function $g(z), z \in \mathbb{Z}$ has a global minimum at $z^{*}$ if $g\left(z^{*}\right) \leq g(z)$ for all $z$. Ponstein [4] considers $f(x)$ to be a real, continuous, and differentiable scalar function with continuous first partial derivatives, of the vector $X$, which is contained in a convex region $R$ of $\mathrm{n}$-dimensional Euclidean space. He then defines a function to be strictly quasi-convex if

$$
f\left(x_{2}\right)<f\left(x_{1}\right) \Rightarrow f\left[\lambda x_{1}+(1-\lambda) x_{2}\right]<f\left(x_{1}\right), 0<\lambda<1, x_{1}, x_{2} \in R .
$$

Kumin [3] extended this definition to give.

Definition 5: A function $g(z), z \in \mathbb{Z}$ is integer strictly quasi-convex if for any two points $z^{1}$ and $z^{2}>z^{1}$ we have the following:

Case I: $g\left(z_{1}\right)<g\left(z_{2}\right)$ implies $g(j)<g\left(z_{2}\right)$ for $j=z_{1}+1, z_{1}+2, \cdots, z_{2}-1$.

Case II: $g\left(z_{1}\right)>g\left(z_{2}\right)$ implies $g(j)<g\left(z_{1}\right)$ for $j=z_{1}+1, z_{1}+2, \cdots, z_{2}-1$.

Ponstein [4] also showed that any local minimum of a strictly quasi convex function is also a global minimum, and Kumin [3] extended his result to show that any local minimum of an integer strictly quasi convex function is also a global minimum.

Now consider a function $f(k, w)$ where $k, w \in \mathbb{Z}$. Also, define $g(k)=f\left(k, w_{k}^{*}\right)$ where $f\left(k, w_{k}^{*}\right)=\min _{w} f(k, w)$.

Theorem 1: Assume that $f(k, w)$ is integer strictly quasi-convex in $w$ for all $k$ and that $g(k)$ is integer strictly quasi-convex in $k$. Then, any local minimum of $f(k, w)$ is also a global minimum.

Proof: Let $(j, m)$ be a local minimum of $f(k, w)$ and suppose it is not a global minimum. Then there exists a $(l, v)$ such that

$$
f(j, m)>f(l, v) \geq f\left(l, w_{l}^{*}\right)
$$

Now, since $f(k, w)$ is strictly integer quasi-convex in $w$ for all $k$

$$
\begin{aligned}
& f(j, m)=f\left(j, w_{j}^{*}\right)=g(m) . \text { Thus, } \\
& f\left(j, w_{j}^{*}\right)>f\left(l, w_{l}^{*}\right) \Rightarrow g(j)>g(l)
\end{aligned}
$$

Assume $j>l$. The proof follows similarly if $j>l$. From Definition 5 , since $g(n)$ is integer strictly quasi convex, then $g(j)>g(l) \Rightarrow g(r)<g(j)$ for 
$r=j+1, j+2, \cdots, l-1$. In particular, for $r=j+1$ we have

$g(j+1)<g(j) \Rightarrow f\left(j+1, w_{j+1}^{*}\right)<f\left(j, w_{j}^{*}\right)=f(j, m)$. But this contradicts the assumption that $(j, m)$ is a local minimum.

Yüceer [5] defines a function to be discretely convex as follows: Let $S$ be a subspace of a discrete $n$-dimensional space. A function $f: S \rightarrow R$ is defined to be discrete convex, if for all $x, y \in S$, and $\alpha>0$, we have $\alpha f(x)+(1-\alpha) f(y) \succcurlyeq \min _{u \in N(z)} f(u)$ where $z=\alpha x+(1-\alpha y)$ and $N(z)=\{u \in S:\|u-z\|<1\}, \quad z=\alpha x+(1-\alpha y)$ and $\|u\|=\max _{1 \leq i \leq n}\left\{\left|u_{i}\right|\right\}$

He then considers the following example

$$
f(i, j)=25(j-i)^{2}+\frac{1}{4}(2-i)^{2} \text { where } i, j \in \mathbb{Z}
$$

Yüceer then shows that $f(i, j)$ is not discretely convex. Below, we show that any local minimum of $f$ is also a global minimum although it is not a discretely convex function.

We first show that $f(i, j)$ is integer strictly quasi-convex in $j \forall i$. According to Definition $2, \Delta^{2} f_{j}(i, j)=100>0 \Rightarrow f(i, j)$ is integer strictly convex in $j \forall i$.

Extending Ponstein's definitions to functions with an integer variable implies that $f(i, j)$ is also integer strictly quasi convex in $j \forall i$ since it is strictly integer convex. Now consider $g(i) \equiv f\left(i, j_{i}^{*}\right)=\min _{j} f(i, j) . j^{*}$ will be a local minimum of $f(i, j)_{i=\text { constant }}$ if $\Delta f_{j}\left(j^{*}\right) \geq 0$ and $\Delta f_{j}\left(j^{*}-1\right) \leq 0$.

For this function: $j^{*}= \begin{cases}\frac{1}{2}(i-1) & \text { if } i \text { is odd i.e. } i=2 k+1 \\ \frac{i}{2} & \text { if } i \text { is even i.e. } i=2 k\end{cases}$

If $i$ is odd, then $i-1$ is an even integer which is divisible by 2 . If $i$ is even, then $i-1$ is an odd integer and not divisible by 2 . Thus, $i=2 k \Rightarrow i-1=2 k-1$ and $\frac{1}{2}(2 k-1)=k-\frac{1}{2}$ where $k \in \mathbb{Z}$. Then $k-1<k-\frac{1}{2}<k$ where $k=\frac{i}{2}$.

Using Definition 3, and by comparing the values of $f(i, k-1)$ and $f(i, k)$ we find that $j^{*}=k=\frac{i}{2}$ is a local minimum of $f(i, j)_{i: \text { even and constant }}$.

Thus, $g(i)= \begin{cases}\frac{1}{4}(2-i)^{2}+25 & \text { if } i \text { is odd } \\ \frac{1}{4}(2-i)^{2} & \text { if } i \text { is even }\end{cases}$

We now verify that $g(i)$ satisfies Definition 5. Assume that $i$ is an odd integer.

1) For any $i_{2}>i_{1}, g\left(i_{1}\right)<g\left(i_{2}\right) \Rightarrow\left|2-i_{1}\right|<\left|2-i_{2}\right|$. Now, since $i_{2}>i_{1}$ we have two conditions to consider: $i_{1}, i_{2}>0$ or $0, i_{1}<0$.

If $i_{1}>0$ and $i_{2}>0$, then for $k=i_{1}+1, i_{1}+2, \cdots, i_{2}-1$, we have $g(k)<g\left(i_{2}\right)$ which satisfies Case I of Definition 5. If $i_{1}<0$ and $i_{2}>0, k$ can be either positive or negative. If $k>0$, then for $k=i_{1}+1, i_{1}+2, \cdots, i_{2}-1$ we have $g(k)<g\left(i_{2}\right)$ which satisfies Case I of Definition 5 . 
If $k<0$ then $i_{1}<k<0$. So for $k=i_{1}+1, i_{1}+2, \cdots, i_{2}-1$ we have $g(k)<g\left(i_{2}\right)$ since $g\left(i_{1}\right)<g\left(i_{2}\right)$ which also satisfies Case I of Definition 5.

2) For any $i_{2}>i_{1}, g\left(i_{1}\right)>g\left(i_{2}\right) \Rightarrow i_{1}<0$ and $i_{2}<0$. So, for $k=i_{1}+1, i_{1}+2, \cdots, i_{2}-1$, we have $g(k)<g\left(i_{1}\right)$ which satisfies Case 2 of Definition 5. The proof follows similarly if $i$ is an even integer.

1) and 2) show that $g(i)$ satisfies the condition of Definition 5, hence it is a strictly integer quasi-convex function. Thus, according to Theorem 1, any local minimum of $f(i, j)$ is a global minimum.

\section{Conclusion}

A sufficient condition is developed for functions of two integer variables such that any local minimum is also a global minimum. This result is important because many optimization problems in integer programming do not have integer convex functions, but still may satisfy this condition.

\section{Conflicts of Interest}

The authors declare no conflicts of interest regarding the publication of this paper.

\section{References}

[1] Tovey, C. (2002) Tutorial on Computational Complexity. Interfaces, 32, 30-61. https://doi.org/10.1287/inte.32.3.30.39

[2] Bazaraa, M., Sherali, H. and Shetty, C. (1993) Nonlinear Programming, Theory and Algorithms. John Wiley \& Sons, New York.

[3] Kumin, H. (1973) On Characterizing the Minimum of a Function of Two Variables, One of Which Is Discrete. Management Science, 20, 126-129. https://doi.org/10.1287/mnsc.20.1.126

[4] Ponstein, J. (1967) Seven Kinds of Convexity. SIAM Review, 9, 115-119. https://doi.org/10.1137/1009007

[5] Yüceer, U. (2002) Discrete Convexity: Convexity for Functions Defined on Discrete Spaces. Discrete Applied Mathematics, 119, 297-304.

https://doi.org/10.1016/S0166-218X(01)00191-3 